\title{
Chicken Mesenchymal Stem Cells as Feeder Cells Facilitate the Cultivation of Primordial Germ Cells from Circulating Blood and Gonadal Ridge
}

\section{Dongsheng Li, Zhisheng Chen, Shengfeng Chen, Huiqin Ji, Xiaoshu Zhan, Dongzhang Luo, Huina Luo, Bingyun Wang*}

School of Life Science and Engineering, Foshan University, Foshan, China

Email: ^bywang63@163.com, dong614lds@1 26.com

How to cite this paper: Li, D.S., Chen, Z.S., Chen, S.F., Ji, H.Q., Zhan, X.S., Luo, D.Z., Luo, H.N. and Wang, B.Y. (2019) Chicken Mesenchymal Stem Cells as Feeder Cells Facilitate the Cultivation of Primordial Germ Cells from Circulating Blood and Gonadal Ridge. Stem Cell Discovery, 9, $1-14$.

https://doi.org/10.4236/scd.2019.91001

Received: January 11, 2019

Accepted: January 28, 2019

Published: January 31, 2019

Copyright $\odot 2019$ by author(s) and Scientific Research Publishing Inc. This work is licensed under the Creative Commons Attribution International License (CC BY 4.0).

http://creativecommons.org/licenses/by/4.0/

\begin{abstract}
Long-term maintenance of chicken primordial germ cells (PGCs) in vitro has tremendous potential for transgenic chicken production. Feeder cells are essential for the establishment and culture of chicken PGCs in vitro. Buffalo rat liver (BRL) cells are the most commonly used feeder cells for PGCs culture; however, this feeder layers from other animal species usually cause immunogenic contaminations, compromising the potential of PGCs in applications. Therefore, we tested chicken source mensenchymal stem cell (MSCs) derived from bone marrow as feeder cells to further improve PGC culture conditions. MSCs derived from chicken bone marrow have a powerful capacity to proliferate and secrete cytokines. We found chicken primordial germ cells derived from circulating blood (cPGCs) and gonads (gPGCs) can be maintained and proliferated with MSCs feeder layer cells. PGCs co-cultured on MSCs feeder retained their pluripotency, expressed PGCs specific genes and stemness markers, and maintained undifferentiated state. Our study indicated that the xeno-free MSCs-feeders culture system is a good candidate for growth and expansion of PGCs as the stepping stone for transgenic chicken research.
\end{abstract}

\section{Keywords}

Chicken, Primordial Germ Cells, Mensenchymal Stem Cells, Transgenic

\section{Introduction}

PGCs are progenitors of germ cells and play an important role in the production of early embryonic germ cells [1] [2]. This unique physiological and develop- 
mental characteristic makes the PGCs valuable as starting cells in producing transgenic chickens [3] [4]. For sustained maintenance in vitro, PGCs isolated from circulating blood at stages $14-17$ and gonadal ridge at stage 28 could be cultured on a feeder layer of Buffalo rat liver (BRL) cells, Mouse Embryonic Fibroblast (MEF) cells, or Sandoz inbred mouse-derived thioguanine-resistant and ouabain-resistant (STO) cells with basic fibroblast growth factor (bFGF) and stem cell factor (SCF) supplementation [5] [6] [7] [8]. Those culture systems use xeno-animal cells as a feeder layer, which may carry the risk of a cross-transfer of pathogens from other animals. The potential risk of cross-species exposure to rodent pathogens and gene products hampers the application of PGCs [9]. Therefore, the development of a chicken-source feeder layer is greatly needed. Chicken embryo fibroblast cells (CEFs) were used to support the growth of PGCs in our earlier study. However, the limited survival time, fewer passage number, and slower proliferation rate of the CEF-feeder increased uncertainty and instability in the process of cultivating the PGCs [10].

MSCs are multipotent stromal cells and can be isolated from different tissues such as bone marrow, umbilical cord, placenta, muscle, adipose tissue, and liver, and can replicate as undifferentiated cells in vitro [11]-[16]. The powerful function of cytokines secretion and proliferation capacity of MSCs are desired characteristics of an effective feeder layer [9]. Previous studies have shown that an MSC-feeder fully supports prolonged human embryonic stem cells (hESCs), human induced pluripotent stem cells (hiPSCs), and mouse embryonic stem cells (mESCs) expansion, and maintained their pluripotency and normal diploid karyotype [17] [18] [19]. We therefore sought to determine whether chicken bone marrow MSCs could be used as feeder layers to support PGC proliferation. In this study, we prove that MSCs derived from bone marrow can replace CEFs to support the generation and propagation of PGCs.

\section{Materials and Methods}

\subsection{Experimental Animals and Chicken Embryos}

The care and experimental use of chickens and fresh fertilized eggs was approved by Nanhai Chicken Breeding Co., Ltd. in Foshan Guangdong. Ephedra chickens were maintained according to the standard management program at the Laboratory Animal Centre, Foshan University, China. Chicken Embryos were incubated in a rotary egg incubator (Rcom PRO 20 , Korea) at $38^{\circ} \mathrm{C}$ and $55 \%$ humidity, with rocking at an angle of $90^{\circ}$ every $1 \mathrm{~h}$ for the following test.

\subsection{Isolation and Culture of Chicken MSCs}

Bone marrow from the femur and tibia of 1- to 10-day-old ephedra chickens was collected by inserting a syringe needle into one end of the bone and washing out with knockout Dulbecco's modified Eagle's medium (KO-DMEM, Gibco, USA). The bone marrow cell suspension was centrifuged at $1000 \mathrm{rpm}$ for $5 \mathrm{~min}$, and then the top fat impurities were removed. The bottom cells were collected and 
washed 3 times with PBS, then re-suspended and plated into a culture plate with complete medium containing KO-DMEM, 7.5\% fetal bovine serum (FBS, Hyclone, Australia), 2.5\% chicken serum (CS, Hyclone, Australia), $2 \mathrm{mM} \mathrm{L-glutamine}$ (Invitrogen), $2 \mathrm{~m}$ MGlutaMAX-I Supplement (Invitrogen), $10 \mathrm{ng} / \mathrm{ml}$ human bFGF (Peprotech, USA), and $10^{4} \mathrm{IU} / \mathrm{mL}$ penicillin/streptomycin (Gibco, USA). The cells were cultured in a $\mathrm{CO}_{2}$ incubator maintained at $37^{\circ} \mathrm{C}$ in an atmosphere of $5 \% \mathrm{CO}_{2}$ in air with $60 \%-70 \%$ relative humidity. The medium was first changed after $24 \mathrm{~h}$, and then once every 3 days. When primary cultures reached $80 \%$ confluence, the cultured MSCs were subcultured by dissociating cells using $0.25 \%$ trypsin containing $0.02 \%$ EDTA.

\subsection{Reverse Transcription PCR of Chicken MSCs}

Chicken MSCs at different passages were collected and total RNA extracted using a Trizol kit (Takara, China). The total RNA was subjected to reverse transcription with random primers and M-MLV enzyme (Takara, China). Transcribed products used to amplify target genes segment with the primers in Table 1. PCR amplification was carried out using the Premix $\mathrm{Taq}^{\mathrm{Tm}}$ version 2.0 plus dye (Takara, China) under the following conditions: denaturation at $95^{\circ} \mathrm{C}$ for 5 min followed by 35 cycles at $94^{\circ} \mathrm{C}$ for $30 \mathrm{~s}, 50^{\circ} \mathrm{C}-60^{\circ} \mathrm{C}$ for $30 \mathrm{~s}$ and $72^{\circ} \mathrm{C}$ for 45 s, followed by $7 \mathrm{~min}$ of final extension at $72^{\circ} \mathrm{C}$. Amplified products were analyzed using $1.5 \%$ agarose gel electrophoresis.

\subsection{Immunocytochemistry of Chicken MSCs}

The Chicken MSCs were fixed in 3.7\% paraformaldehyde solution for $30 \mathrm{~min}$, washed three times with PBS containing 5\% (v/v) fetal bovine serum, and blocked with blocking buffer consisting of PBS containing 10\% (v/v) fetal bovine serum, for $30 \mathrm{~min}$. Cells were then washed three times (10 min per wash) with PBS containing 5\% FBS, and incubated with primary antibodies against CD 29 and CD 44 (1:200, Santa Cruz, CA) at $4^{\circ} \mathrm{C}$ overnight. Afterwards, the primary antibody was removed and cells were washed three times (10 min per wash) with PBS containing 5\% FBS. Cells were then incubated with secondary antibodies (1:500, Santa Cruz, CA) labeled with FITC at room temperature in the dark for 1 h. The samples were finally incubated with DAPI (Invitrogen) for $5 \mathrm{~min}$, protected from light, and analyzed under a fluorescence microscope (Olympus).

\subsection{Differentiation of Chicken MSCs}

At the third passage, when the confluence of cells reached $80 \%$, the medium was removed and the cells were washed with PBS 3 times. The adipogenesis differentiation induction medium (ADM, Biowit, China) and osteogenic differentiation induction medium (ODM, Biowit, China) were added to the induced group and control cells cultured in complete medium. The media was completely replaced every 3 - 4 days Adipogenic potential was assessed by Oil Red O staining, and osteogenic capacity was determined by alizarin red staining after inducting for 2 
Table 1. PCR primer sequences.

\begin{tabular}{|c|c|c|}
\hline Gene & Sequence $\left(5^{\prime}-3^{\prime}\right)$ & Length, bp \\
\hline \multirow{2}{*}{$\mathrm{CD} 29$} & GAACGGACAGATATGCAACGG & \multirow{2}{*}{300} \\
\hline & TAGAACCAGCAGTCACCAACG & \\
\hline \multirow{2}{*}{ CD34 } & GTGCCACAACATCAAAGACG & \multirow{2}{*}{239} \\
\hline & GGAGCACATCCGTAGCAGGA & \\
\hline \multirow{2}{*}{ CD44 } & CATCGTTGCTGCCCTCCT & \multirow{2}{*}{290} \\
\hline & ACCGCTACACTCCACTCTTCAT & \\
\hline \multirow{2}{*}{ CD71 } & CCCAGGCTTCCCTTCGT & \multirow{2}{*}{305} \\
\hline & GGGCTCCAATCACAACATAC & \\
\hline \multirow{2}{*}{ PPAR- $\gamma$} & CTGTCTGCGATGGATGAT & \multirow{2}{*}{199} \\
\hline & AATAGGGAGGAGAAGGAG & \\
\hline \multirow{2}{*}{ FAS } & GACCCACCACGTCCCTGACATTG & \multirow{2}{*}{193} \\
\hline & GGTTTCGTAGGCTCCTCCCATCC & \\
\hline \multirow{2}{*}{ OSTEOPONTIN } & CTTGCTCGCCTTCACCAC & \multirow{2}{*}{227} \\
\hline & CTGTCTGCGATGGATGAT & \\
\hline \multirow{2}{*}{ BMP2 } & CGCTTACGCTGTTTGTGTTTCG & \multirow{2}{*}{192} \\
\hline & GGTGGAGGTGGTTCACTTGGA & \\
\hline \multirow{2}{*}{ NANOG } & TGGTTTCAGAACCAACGAATGAAG & \multirow{2}{*}{180} \\
\hline & TGCACTGGTCACAGCCTGAAG & \\
\hline \multirow{2}{*}{ POUV } & GTTGTCCGGGTCTGGTTCT & \multirow{2}{*}{189} \\
\hline & GTGGAAAGGTGGCATGTAGAC & \\
\hline \multirow{2}{*}{ SOX2 } & GAAGATGCACAACTCGGAGATCA & \multirow{2}{*}{100} \\
\hline & GAGCCGTTTGGCTTCGTCA & \\
\hline \multirow{2}{*}{$\mathrm{CVH}$} & GTCTGCCTGTGCAGCATGACATTG & \multirow{2}{*}{202} \\
\hline & CTTTGCCCAAAGATGCCAGGAACTC & \\
\hline \multirow{2}{*}{ DAZL } & CGTCAACAACCTGCCAAGGA & \multirow{2}{*}{540} \\
\hline & TTCTTTGCTCCCCAGGAACC & \\
\hline \multirow{2}{*}{ ACTB } & ATTGTCCACCGCAAATGCTTC & \multirow{2}{*}{113} \\
\hline & AAATAAAGCCATGCCAATCTCGTC & \\
\hline
\end{tabular}

weeks. Adipogenic and osteoblast specific genes were further detected using RT-PCR.

\subsection{Feeder Cell Preparation}

The chicken MSCs and embryo fibroblast cells at passage 3 - 8 were seeded in 6 -well plates. When cultures reached $80 \%$ confluence the medium was removed, and cells were treated with $10 \mathrm{ug} / \mathrm{ml}$ Mitomycin C for $2 \mathrm{~h}$. Afterwards, they were washed five times with PBS, and then the mitotic inactivated cells were seeded in feeder cell medium to achieve a confluent layer.

\subsection{Cultivation of Primordial Germ Cells}

Blood cells, including cPGCs, were isolated from the vasculature system of stage 14 - $16 \mathrm{HH}$ ephedra chicken embryos and cultured on dishes containing feeder cells pretreated with Mitomycin C in KO-DMEM, 7.5\% FBS, 2.5\% chicken serum (CS, Hyclone, Australia), 2 mM GlutaMAX-I Supplement (Invitrogen), $1 \times$ nucleosides (Millipore, CA), $1 \times$ nonessential amino acids, $\beta$-mercaptoethanol, 
and combinations of the following growth factors: $5 \mathrm{ng} / \mathrm{ml}$ human LIF (Peprotech, USA), $5 \mathrm{ng} / \mathrm{ml}$ human SCF (Peprotech, USA), and $10 \mathrm{ng} / \mathrm{ml}$ human bFGF (Peprotech, USA).

The embryos at stage $28 \mathrm{HH}$ and incubated for 5.5 days were retrieved and rinsed three times with PBS. Gonadal ridges were isolated by medial section of the abdomen with sharp tweezers under a stereomicroscope (SZX16, Olympus). Gonadal tissue was dissociated into single cells with $0.25 \%$ trypsin containing $0.02 \%$ EDTA, and then washed with PBS and centrifuged at $1000 \mathrm{rpm}$ for $5 \mathrm{~min}$. The primary gonadal cells, including gPGCs and somatic cells, were re-suspended in complete medium, the same as the cPGCs, and the moved to a $35 \mathrm{~mm}$ dish without feeder cells. The somatic cells adsorbed on the surface of the dish completely in $24 \mathrm{~h}$, and then suspended gPGCs were moved to a new dish containing mitotic inactivated feeder cells.

\subsection{Chicken PGCs Specific Gene Expression}

The methods of RNA isolation and Reverse Transcription PCR were previously described in Reverse Transcription PCR of Chicken MSCs above. RT-PCR analysis was performed to determine the expression level of the PGCs-specific genes, including Nanog, PouV, Sox2, Cvh, and Dazl. Primer sequences are summarized in Table 1.

\subsection{Immunofluorescent Detection of PGC Surface Markers}

Three plates cultured cPGCs and gPGCs were prepared to detect Chicken PGC surface markers SSEA-1 and Dazl using anti-SSEA-1 (1:200, Santa Cruz, CA) and anti-Dazl (1:200, Santa Cruz, CA) antibodies. The specific steps are the same as in Immunocytochemistry of Chicken MSCs, given above.

\subsection{Proliferation Assays of PGCs}

The cPGCs and gPGCs at passage 2 that dissociated to single cells were seeded in 12-well plates and cultured with MSCs-feeder cells, CEF-feeder cells, and withoutfeeder. After culturing for 3 days, the PGCs were moved to 96-well plates in order to detect the efficiency of cell proliferation using Cell Counting Kit-8 (CCK-8, Beyotime, China). After treatment with CCK-8 in a $\mathrm{CO}_{2}$ incubator maintained at $37^{\circ} \mathrm{C}$, cells were moved to determine the absorbance values of $\mathrm{OD}$ 450 using a Microplate Reader (Thermo).

\section{Results}

\subsection{Isolation, Culture, and Morphology of Chicken MSCs}

The primary cells were isolated from bone marrow of 1 to 10 day old ephedra chickens and cultured in the presence of bFGF. After 7 days of growth, most of the blood cells were dead and most MSCs were attached to the culture plate. The cells expanded easily and exhibited fibroblast-like morphology. Approximately 10 days later, the cells grew to $80 \%-90 \%$ confluence and were passaged for the 
first time. After 3 passages, the cultures were very pure and displayed a unique vortex shape. There were no obvious morphological differences among different passages and cellular morphology remained stable after serial passages (Figures 1(A)-(D)). Eventually, as passage numbers increased, the cells showed signs of slowed proliferation (Figure 1(E)) and senescence. Moreover, cell fusion and cavitation began to appear on the 22nd passage.

\subsection{RT-PCR Analysis of Chicken MSCs}

RT-PCR experiments showed that chicken MSCs expressed the pluripotent stem cell marker, Nanog, and mesenchymal stem cell markers, CD29, CD44, and CD71. All the above cell markers are positive, and the hematopoietic blood stem cell marker, CD34, was negative (Figure 2).

\subsection{Immunofluorescence of Chicken MSCs}

Specific marker proteins for chicken MSCs were detected through immunofluorescence staining. Expression of CD29 and CD44 was observed in chicken MSCs (Figure 3).

\subsection{Differentiation of Chicken MSCs}

Adipogenic Differentiation of chicken MSCs. After induction with adipogenic inducers for 2 weeks, the chicken MSCs changed gradually from fibroblast-like cells to flattened cells (Figure 4(A)), and many lipid droplets accumulated in them. The adipogenic differentiated MSCs were visualized by staining with Oil Red $\mathrm{O}$ on the $15^{\text {th }}$ day. The cellular staining was positive and the multiple lipid vacuoles in differentiated cells were stained red (Figure 4(B)). The RT-PCR assay indicated that after induction with adipogenic inducers, the adipocyte specific genes, fatty acid synthetase (FAS), and peroxisome proliferator-activated receptor $\gamma$ (PPAR- $\gamma$ ) were present (Figure $4(C)$ ).

Osteogenic Differentiation of chicken MSCs. After incubation in medium for 15 days, chicken MSCs showed obvious morphological changes. Tightly packed colonies forming nodule-like structures were observed. Deposition of calcium in these cells was shown by staining with alizarin red (Figure 4(E)). RT-PCR assay indicated that, after incubation with ODM, the osteogenic specific genes, osteopontin and bone morphogenetic protein (BMP2), were detected in the induced group and not in the control group (Figure $4(\mathrm{~F})$ ).

\subsection{Culture of Chicken PGCs}

cPGCs from chicken embryos at stages 14 - 15 were isolated and cultured on MSCs-feeders. After 7 - 14 days of growth, most of the blood cells had disappeared, and PGC colonies had formed and were loosely attached on culture MSCs-feeders (Figure 5(A)). gPGCs from chicken embryos at stage 28 were plated down and sporadically found in the mixed population of gonadal cells. The PGC colonies formed in the primary culture and after several passages, the 

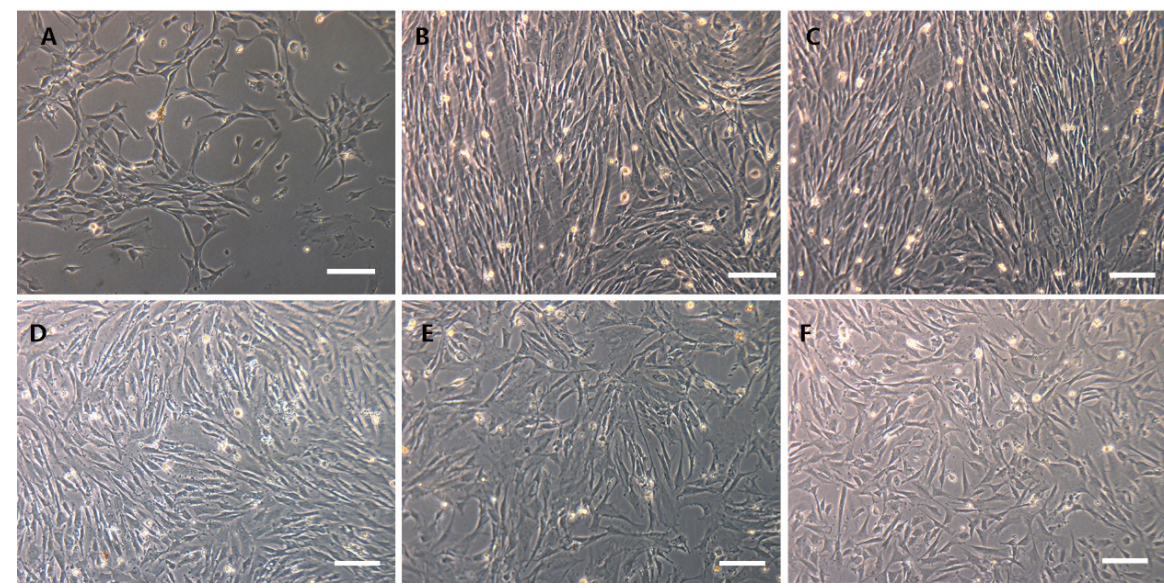

Figure 1. Cell morphology at different stages along with chicken MSC culture. (A) Chicken MSCs on day 6 of primary culture. MSCs cell-cloning began to form and most of the other bone marrow cells died and disappeared; (B) chicken MSCs at P1. Cells increased quickly and aggregated in certain areas; (C) chicken MSCs at P3. MSCs were very pure and homogeneous with a typical long spindle-shape; (D) chicken MSCs at P8. The culture displayed a unique vortex shape; (E) chicken MSCs at P15. The cells showed signs of slowed proliferation; (F) thawed chicken MSCs. MSCs can be successfully cryopreserved. Cellmorphology and proliferation ability were maintained after cryopreservation. Scale bar $=100 \mu \mathrm{m}$.

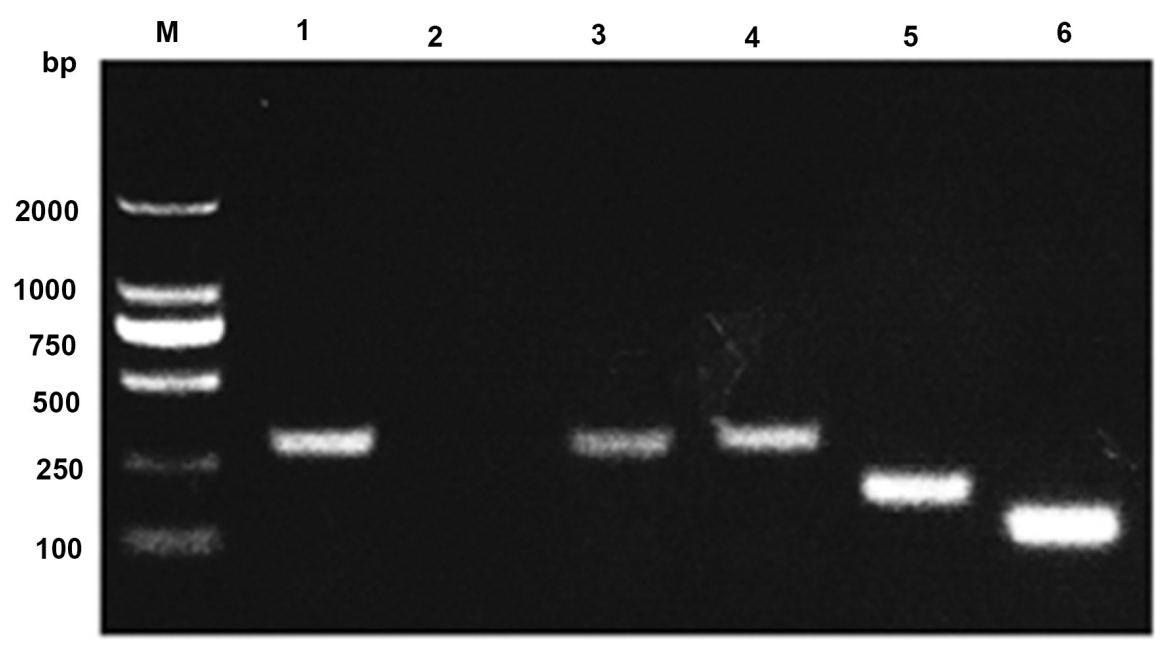

Figure 2. RT-PCR analysis of chicken MSCs. (M) DL2000 DNA Maker; (1) CD29; (2) CD34; (3) CD44; (4) CD71; (5) Nanog; (6) beta actin.

other gonadal cells were dead. Next, the pure gPGCs were cultivated on MSCs-feeders (Figure 5(B)). The PGCs were subcultured by trypsin every 4 - 5 days.

\subsection{Identification of Chicken PGCs Cultured on MSCs-Feeders}

Immunocytochemical analysis was performed to characterize cultured PGCs in detail. These cPGCs and gPGCs were positive for the chicken PGC markers SSEA-1 and DAZL (Figure 6). To examine the PGC-specific gene expression, 

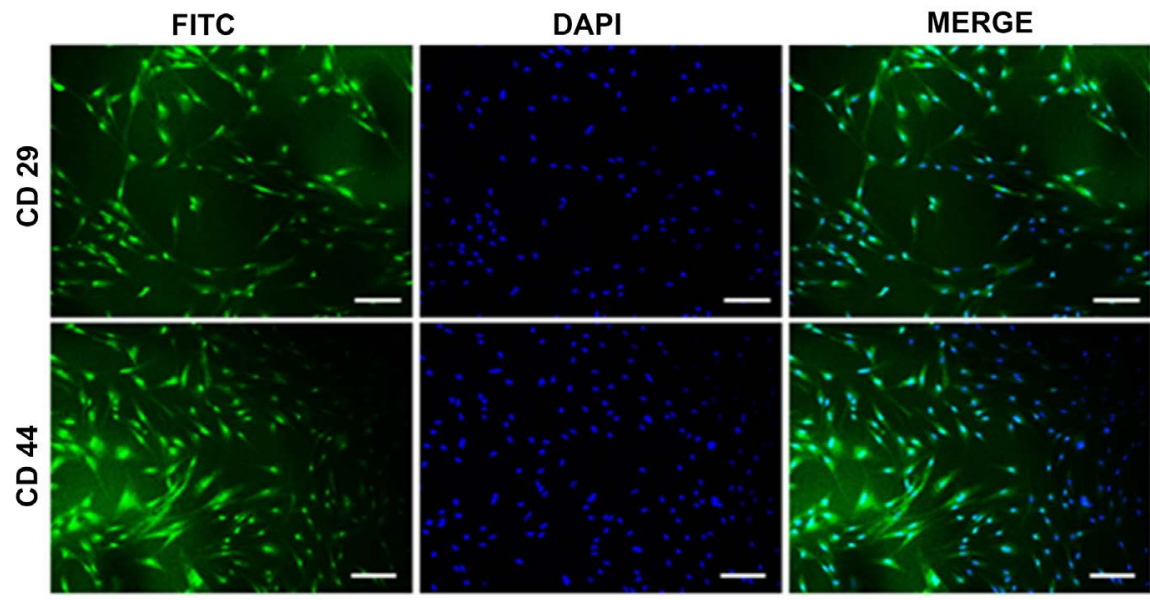

Figure 3. Immunolocalization of surface markers in chicken MSCs. The surface markers of CD29 and CD44 were positive-staining and the nuclei were stained with DAPI. Scale bar $=100 \mu \mathrm{m}$.
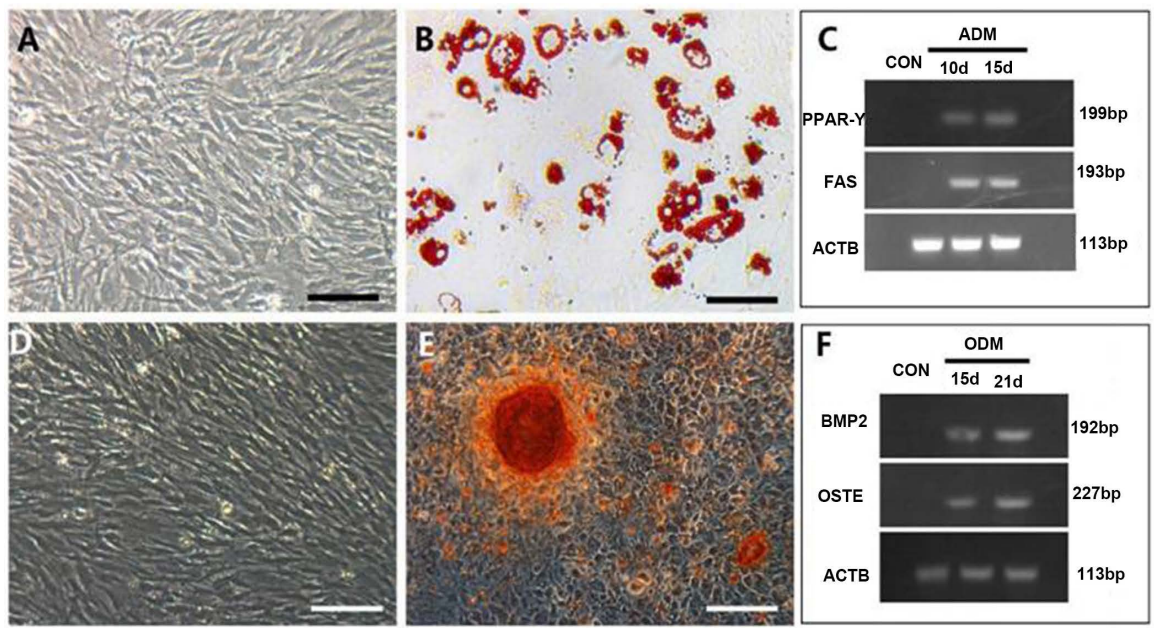

Figure 4. Adipogenic and osteogenic differentiation of chicken MSCs. (A) control cells of cultured adipogenic differentiation in complete medium did not change in morphology; (B) after induction for 15 days, MSCs became oblate and formed many intracellular lipid droplets. Lipid droplets were stained with oil red O; (C) expression of adipocyte-specific genes FAS and PPAR- $\gamma$ was detected by RT-PCR in the induced group after induction for 15 days. The beta actin (ACTB) gene served as the internal control; (D) control cells of cultured osteogenic differentiation in complete medium did not change in morphology; (E) after induction for 15 days, the cells changed from a fusiform shape to a triangular shape, and calcified nodules increased in number and became larger. The nodules were observed using alizarin red staining; (F) expression of adipocyte-specific genes BMP2 and osteopontin was detected by RT-PCR in the induced group after induction for 15 days and 21 days. The beta actin (ACTB) gene served as the internal control. Scale bar $=100$ $\mu \mathrm{m}$.

RT-PCR analysis was performed (Figure 7). Expression of Nanog, PouV, and Sox2, three genes known to be expressed inpluripotent stem cell, were detected. The germ cell specific genes Cvh and Dazl were also expressed in cultured cPGCs and gPGCs. 

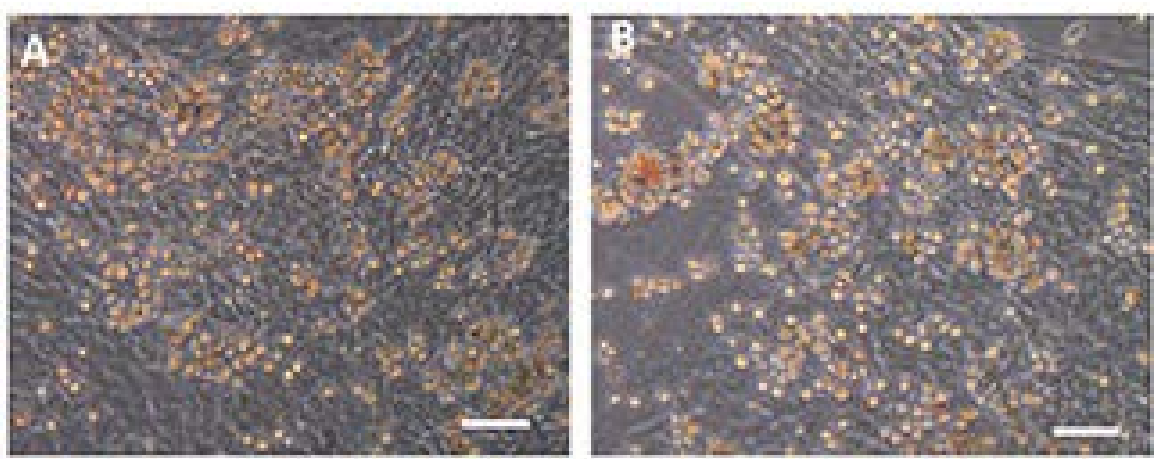

Figure 5. Morphology of PGCs cultured in vitro. (A) Passage 3 cPGCs isolated from embryonic circulating blood; (B) passage 3 gPGCs isolated from the gonadal ridge. Both the cPGCs in (A) and the gPGCs in (B) were cultured on MSC feeder cells derived from chick bone marrow. Scale bar $=100 \mu \mathrm{m}$.
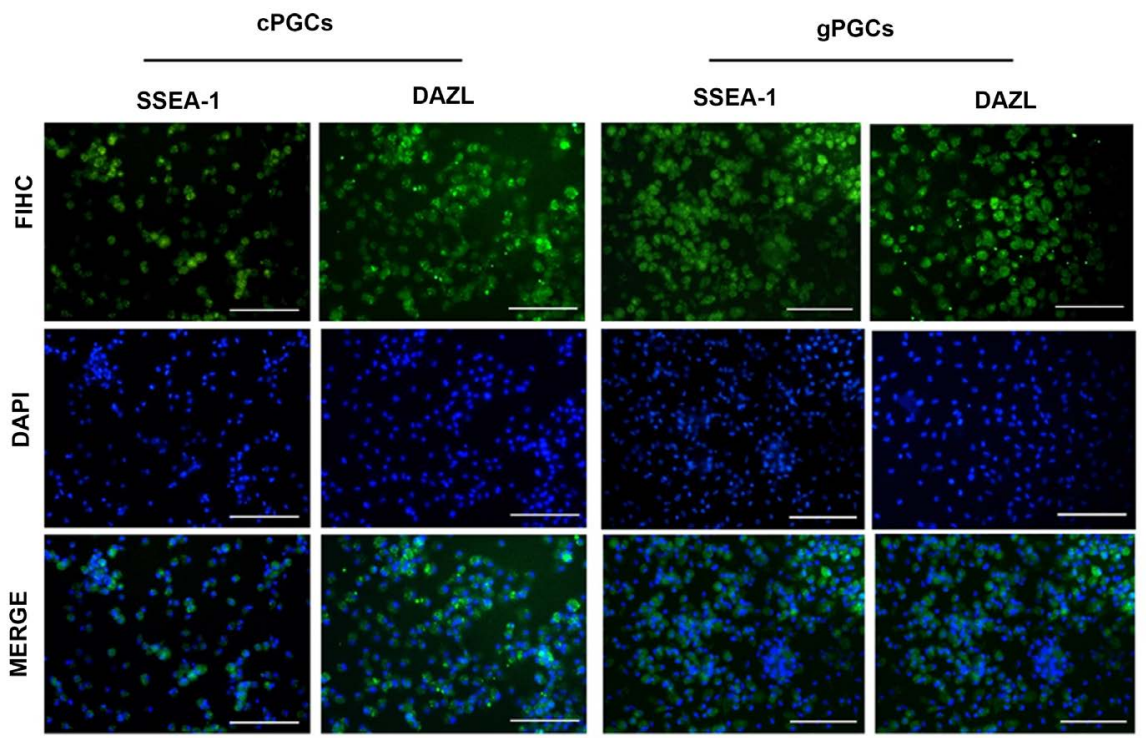

Figure 6. Immunofluorescence staining of PGCs. The specific markers (SSEA-1 and DAZL) of cPGCs and gPGCs were positive-staining, and nuclei were stained with DAPI. Scale bar $=100 \mu \mathrm{m}$.

\subsection{Proliferation of Chicken PGCs Cultured on Different Feeders}

The proliferation activity of PGCs on MSCs- and CEFs-feeder cells was detected using CCK-8. The results showed that the feeder cells can promote proliferation of cPGCs and gPGCs in vitro, and that MSCs-feeder cells were better at enhancing the proliferation of PGCs rather than CEFs-feeder cells (Figure 8).

\section{Discussion}

PGCs are precursor cells of spermatozoa and ova [20]. As a kind of pluripotent stem cell, chicken PGCs have a similar morphology and multilineage potential to ESCs, which can be cultured and subcultured in vitro and maintain their undifferentiated state and pluripotency, under certain conditions [21] [22]. A feeder-free culture system of chicken PGCs has not been established, and the 


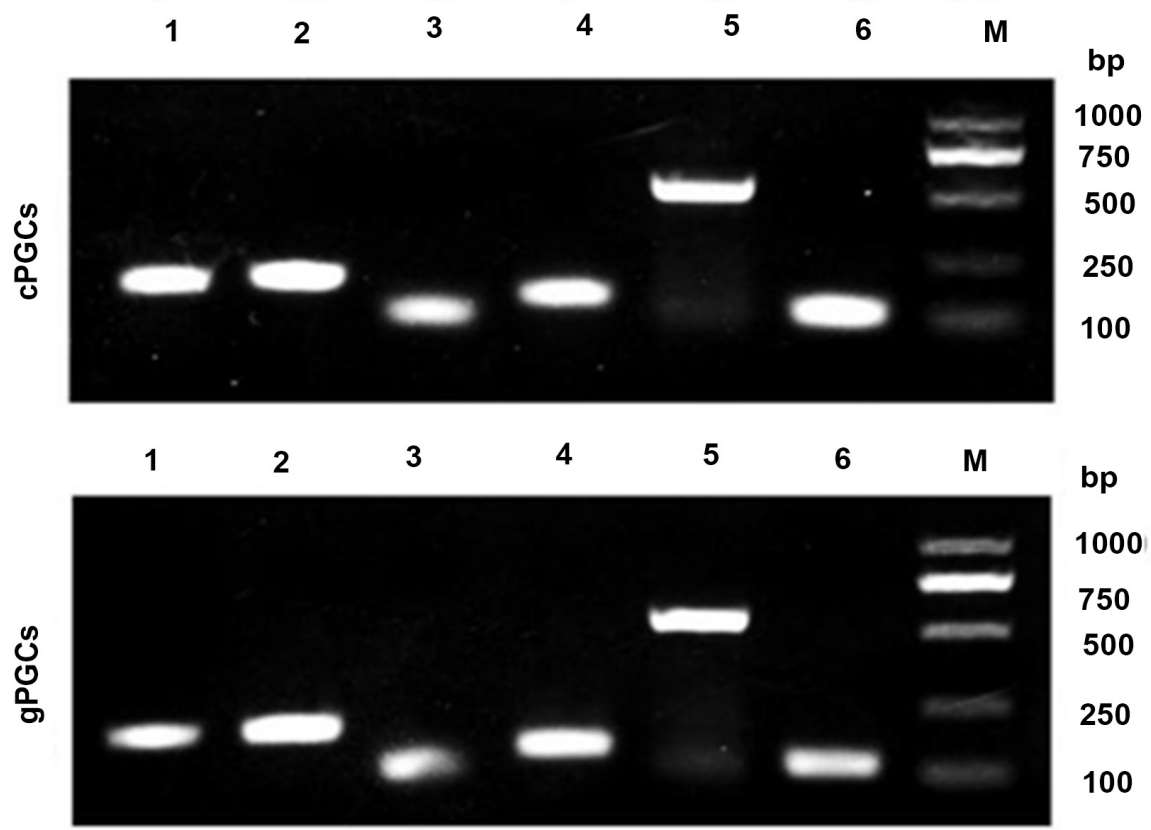

Figure 7. RT-PCR analysis of chicken MSCs. (M) DL2000 DNA Maker; (1) Nanog; (2) PouV; (3) Sox2; (4) Cvh; (5) Dazl; (6) beta actin.

cPCGs

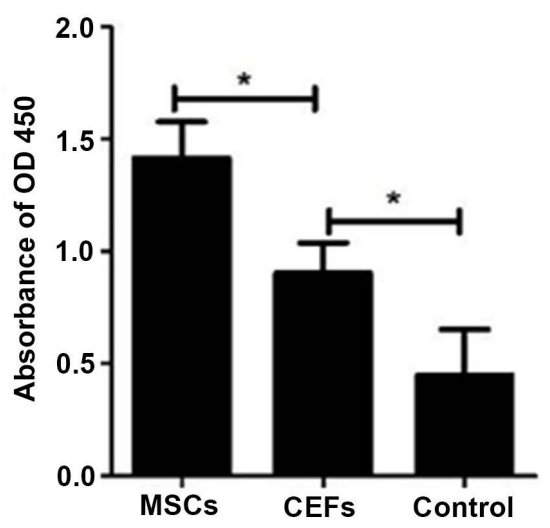

gPCGs

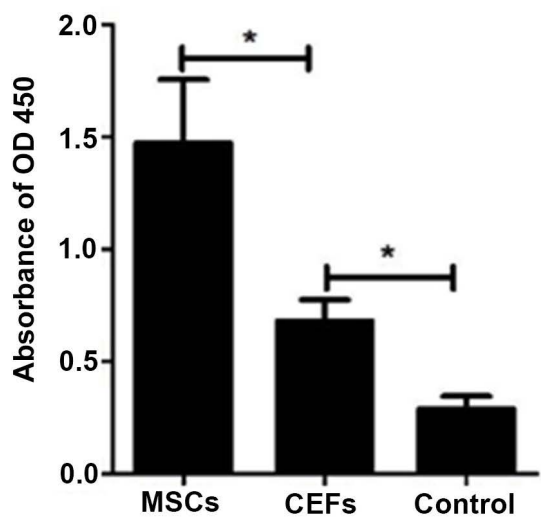

Figure 8. Proliferation of chicken PGCs cultured on different feeders cells. The absorbance values of OD 450 of cPGCs and gPGCs co-cultured with MSCs-feeder cells, CEF-feeder cells, and without feederS. Values are shown as mean \pm standard deviation; $\mathrm{n}$ $=5 .{ }^{*} \mathrm{P}<0.05$.

proliferation in vitro of chicken PGC culture depends on the feeder cells being present [23] [24]. Feeder cells not only prevent the PGCs from attaching to the plastic dishes, but can also secrete a variety of growth factors inhibiting the differentiation and promoting the proliferation of PGCs in vitro [5]. Normally, MEF, BRL, and STO have been applied extensively to support self-renewal and proliferation of chicken PGCs for in vitro cultures, but the traditional cross species animal origin feeders unavoidably increase the risk of releasing animal materials and unknown pathogens [25] [26] [27]. Therefore, development of chicken source feeder cells is greatly needed. This study is the firstly attempt to 
use chicken source MSCs-feeder cells as a xeno-free culture system to maintain chicken PGC proliferation.

MSCs are multipotent stromal cells from the mesoderm and can be isolated from various sources such as bone marrow, adipose tissue and others [28] [29] [30]. Chicken MSCs are advantageous in that they are easy to collect from the bone arrow cavities of both embryonic and adult ages, with simple and well-established protocols for in vitro collection [15]. MSCs derived from bone marrow have been shown to be able to differentiate into adipocytes, osteocytes, chondrocytes, and neural-like cells, among others [16]. These cells also have the ability to produce cytokines and growth factors that support and regulate haematopoiesis, proliferation, differentiation, and immunoregulation [31]. Previous studies have demonstrated thathuman embryonic stem cells (hESCs) [32] [33], human induced pluripotent stem cells (hiPSCs) [9] [34], andhuman hematopoietic stem cell (hHSCs) [17] [35] had been continuously co-cultured with MSCs-feeder in vitro.

In our study, a xeno-free feeder layer system was developed for prolonged expansion of chicken PGCs in culture with a bone marrow MSCs-feeder. Chicken cPGCs and gPGCs showed clonal growth when co-cultured on a mitomycin C treated MSCs feeder layer. The positive expression of NANOG, SOX2, POUV, DAZL, and CVH was apparent, implying that the pluripotency andgenitality of PGCs was not primarily changed after prolonged cultured with an MSCs feeder. The different levels of cell proliferation of PGCs co-cultured on a MSCs- feeder layer, a CEFs-feeder layer, and a feeder-free layer was determined by cck-8 kit. The results indicated that feeder cells can significantly increase cell proliferation, and that the MSCs-feeder layer performed better than the CEFs-feeder layer. Both the cPGCs and gPGCs co-cultured on MSCs-feeder layers expressed PGCs specific surface and pluripotent gene expression and had a good state of growth. An MSCs-feeder layer is therefore more suitable for PGCs cultivation in vitro rather than a CEFs-feeder layer. It is possible that the MSCs-feeder layer provids a more suitable microenvironment for PGCs.

This study confirmed that chicken MSCs are a more efficient source of chicken feeder cells, capable of maintaining the growth of PGCs and their undifferentiated state. The results provide a new feeder system to deal with the current problems associated with chicken PGC cultivation in vitro, and show its potential future application.

\section{Conclusion}

In conclusion, we demonstrated that MSCs-feeder could improve the in vitro expansion of chicken PGCs and generate a greater number of primitive cells during this expansion. Our results provide insight into the potential use of MSCs-feeders in vitro to aid the expansion of chicken PGCs cultures.

\section{Acknowledgements}

The authors report no conflicts of interest. The authors alone are responsible for 
the content and writing of the paper.

\section{Conflicts of Interest}

The authors declare no conflicts of interest regarding the publication of this paper.

\section{References}

[1] Motono, M., Ohashi, T., Nishijima, K. and Iijima, S. (2008) Analysis of Chicken Primordial Germ Cells. Cytotechnology, 57, 199-205.

https://doi.org/10.1007/s10616-008-9156-x

[2] Petitte, J.N., Liu, G. and Yang, Z. (2004) Avian Pluripotent Stem Cells. Mechanisms of Development, 121, 1159-1168. https://doi.org/10.1016/j.mod.2004.05.003

[3] Macdonald, J., Glover, J.D., Taylor, L., Sang, H.M. and McGrew, M.J. (2010) Characterisation and Germline Transmission of Cultured Avian Primordial Germ Cells. PLoS ONE, 5, e15518. https://doi.org/10.1371/journal.pone.0015518

[4] Kim, J.N., Park, T.S., Park, S.H., et al. (2010) Migration and Proliferation of Intact and Genetically Modified Primordial Germ Cells and the Generation of a Transgenic Chicken. Biology of Reproduction, 82, 257-262.

https://doi.org/10.1095/biolreprod.109.079723

[5] Song, Y., Duraisamy, S., Ali, J., et al. (2014) Characteristics of Long-Term Cultures of Avian Primordial Germ Cells and Gonocytes. Biology of Reproduction, 90, 1-8. https://doi.org/10.1095/biolreprod.113.113381

[6] Jung, J.G., Kim, D.K., Park, T.S., et al. (2005) Development of Novel Markers for the Characterization of Chicken Primordial Germ Cells. Stem Cells, 23, 689-698. https://doi.org/10.1634/stemcells.2004-0208

[7] Shiue, Y.L., Tailiu, J.J., Liou, J.F., et al. (2009) Establishment of the Long-Term in Vitro Culture System for Chicken Primordial Germ Cells. Reproduction in Domestic Animals, 44, 55-61. https://doi.org/10.1111/j.1439-0531.2007.00990.x

[8] Chen, D., Chen, M., Lu, Z., et al. (2016) Cholesterol Induces Proliferation of Chicken Primordial Germ Cells. Animal Reproduction Science, 171, 36-40. https://doi.org/10.1016/j.anireprosci.2016.05.011

[9] Zou, Q., Wu, M., Zhong, L., et al. (2016) Development of a Xeno-Free Feeder-Layer System from Human Umbilical Cord Mesenchymal Stem Cells for Prolonged Expansion of Human Induced Pluripotent Stem Cells in Culture. PLoS ONE, 11, e149023.

[10] Kong, L.L., Qiu, L.L., Guo, Q.X., et al. (2018) Long-Term in Vitro Culture and Preliminary Establishment of Chicken Primordial Germ Cell Lines. PLoS ONE, 13, e196459. https://doi.org/10.1371/journal.pone.0196459

[11] Joerger-Messerli, M.S., Marx, C., Oppliger, B., et al. (2016) Mesenchymal Stem Cells from Wharton's Jelly and Amniotic Fluid. Best Practice \& Research: Clinical Obstetrics \& Gynaecology, 31, 30-44. https://doi.org/10.1016/j.bpobgyn.2015.07.006

[12] Zhu, W., Sun, H. and Lyu, G. (2015) Advances in the Mechanism of Mesenchymal Stem Cells in Promoting Wound Healing. Chinese Journal of Burns, 31, 476-478.

[13] Gao, Y., Bai, C., Wang, K., et al. (2014) All-Trans Retinoic Acid Promotes Nerve Cell Differentiation of Yolk Sac-Derived Mesenchymal Stem Cells. Applied Biochemistry and Biotechnology, 174, 682-692. https://doi.org/10.1007/s12010-014-1100-2 
[14] Bai, C., Li, X., Hou, L., et al. (2013) Biological Characterization of Chicken Mesenchymal Stem/Progenitor Cells from Umbilical Cord Wharton's Jelly. Molecular and Cellular Biochemistry, 376, 95-102. https://doi.org/10.1007/s11010-012-1553-y

[15] Bai, C., Hou, L., Ma, Y., et al. (2013) Isolation and Characterization of Mesenchymal Stem Cells from Chicken Bone Marrow. Cell and Tissue Banking, 14, 437-451. https://doi.org/10.1007/s10561-012-9347-8

[16] Khatri, M., O'Brien, T.D. and Sharma, J.M. (2009) Isolation and Differentiation of Chicken Mesenchymal Stem Cells from Bone Marrow. Stem Cells and Development, 18, 1485-1492. https://doi.org/10.1089/scd.2008.0223

[17] Iacono, M.L., Anzalone, R., La Rocca, G., et al. (2017) Wharton's Jelly Mesenchymal Stromal Cells as a Feeder Layer for the ex Vivo Expansion of Hematopoietic Stem and Progenitor Cells: A Review. Stem Cell Reviews and Reports, 13, 35-49. https://doi.org/10.1007/s12015-016-9702-4

[18] Xie, H., Sun, L., Zhang, L., et al. (2016) Mesenchymal Stem Cell-Derived Microvesicles Support ex Vivo Expansion of Cord Blood-Derived CD34 ${ }^{+}$Cells. Stem Cells International, 2016, Article ID: 6493241.

[19] Chang, Y.H., Chu, T.Y. and Ding, D.C. (2017) WNT/Beta-Catenin Signaling Pathway Regulates Non-Tumorigenesis of Human Embryonic Stem Cells Co-Cultured with Human Umbilical Cord Mesenchymal Stem Cells. Scientific Reports, 7, Article No. 41913.

[20] Park, T.S. and Han, J.Y. (2012) Genetic Modification of Chicken Germ Cells. Annals of the New York Academy of Sciences, 1271, 104-109. https://doi.org/10.1111/j.1749-6632.2012.06744.x

[21] Bhuvanalakshmi, G., Arfuso, F., Dharmarajan, A. and Warrier, S. (2014) Multifunctional Properties of Chicken Embryonic Prenatal Mesenchymal Stem Cells-Pluripotency, Plasticity, and Tumor Suppression. Stem Cell Reviews and Reports, 10, 856-870. https://doi.org/10.1007/s12015-014-9530-3

[22] Choi, J.W., Kim, S., Kim, T.M., et al. (2010) Basic Fibroblast Growth Factor Activates MEK/ERK Cell Signaling Pathway and Stimulates the Proliferation of Chicken Primordial Germ Cells. PLoS ONE, 5, e12968.

[23] Tonus, C., Cloquette, K., Ectors, F., et al. (2016) Long Term-Cultured and Cryopreserved Primordial Germ Cells from Various Chicken Breeds Retain High Proliferative Potential and Gonadal Colonisation Competency. Reproduction, Fertility and Development, 28, 628-639. https://doi.org/10.1071/RD14194

[24] Naeemipour, M., Dehghani, H., Bassami, M., et al. (2013) Expression Dynamics of Pluripotency Genes in Chicken Primordial Germ Cells before and after Colonization of the Genital Ridges. Molecular Reproduction and Development, 80, 849-861. https://doi.org/10.1002/mrd.22216

[25] Nandi, S., Whyte, J., Taylor, L., et al. (2016) Cryopreservation of Specialized Chicken Lines Using Cultured Primordial Germ Cells. Poultry Science, 95, 1905-1911. https://doi.org/10.3382/ps/pew133

[26] Lee, H.C., Choi, H.J., Lee, H.G., et al. (2016) DAZL Expression Explains Origin and Central Formation of Primordial Germ Cells in Chickens. Stem Cells and Development, 25, 68-79. https://doi.org/10.1089/scd.2015.0208

[27] Jang, H.J., Seo, H.W., Lee, B.R., et al. (2013) Gene Expression and DNA Methylation Status of Chicken Primordial Germ Cells. Molecular Biotechnology, 54, 177-186. https://doi.org/10.1007/s12033-012-9560-5

[28] Romanov, Y.A., Balashova, E.E., Volgina, N.E., et al. (2015) Optimized Protocol for 
Isolation of Multipotent Mesenchymal Stromal Cells from Human Umbilical Cord. Bulletin of Experimental Biology and Medicine, 160, 148-154. https://doi.org/10.1007/s10517-015-3116-1

[29] Hu, P., Pu, Y., Li, X., et al. (2015) Isolation, in Vitro Culture and Identification of a New Type of Mesenchymal Stem Cell Derived from Fetal Bovine Lung Tissues. Molecular Medicine Reports, 12, 3331-3338. https://doi.org/10.3892/mmr.2015.3854

[30] Bacenkova, D., Rosocha, J., Tothova, T., et al. (2011) Isolation and Basic Characterization of Human Term Amnion and Chorion Mesenchymal Stromal Cells. Cytotherapy, 13, 1047-1056.

[31] Spencer, N.D., Gimble, J.M. and Lopez, M.J. (2011) Mesenchymal Stromal Cells: Past, Present, and Future. Veterinary Surgery, 40, 129-139.

https://doi.org/10.1111/j.1532-950X.2010.00776.x

[32] Soong, Y.K., Huang, S.Y., Yeh, C.H., et al. (2015) The Use of Human Amniotic Fluid Mesenchymal Stem Cells as the Feeder Layer to Establish Human Embryonic Stem Cell Lines. Journal of Tissue Engineering and Regenerative Medicine, 9, E302-E307.

[33] Zhang, K., Cai, Z., Li, Y., et al. (2011) Utilization of Human Amniotic Mesenchymal Cells as Feeder Layers to Sustain Propagation of Human Embryonic Stem Cells in the Undifferentiated State. Cellular Reprogramming, 13, 281-288. https://doi.org/10.1089/cell.2010.0103

[34] Havasi, P., Nabioni, M., Soleimani, M., Bakhshandeh, B. and Parivar, K. (2013) Mesenchymal Stem Cells as an Appropriate Feeder Layer for Prolonged in Vitro Culture of Human Induced Pluripotent Stem Cells. Molecular Biology Reports, 40, 3023-3031. https://doi.org/10.1007/s11033-012-2376-3

[35] Huang, X., Zhu, B., Wang, X., Xiao, R. and Wang, C. (2016) Three-Dimensional Co-Culture of Mesenchymal Stromal Cells and Differentiated Osteoblasts on $\mathrm{Hu}$ man Bio-Derived Bone Scaffolds Supports Active Multi-Lineage Hematopoiesis in Vitro: Functional Implication of the Biomimetic HSC Niche. International Journal of Molecular Medicine, 38, 1141-1151. https://doi.org/10.3892/ijmm.2016.2712 